\title{
Safety oversight of lasers in the U.S. Air Force
}

\author{
M. D. Gifford \\ U.S. Air Force, HQ Air Force Safety Center, USA
}

\begin{abstract}
The United States Air Force is a significant user of lasers in military operations, and this use is growing rapidly. The Air Force has safety oversight processes designed to identify hazards of specific laser systems, communicate those hazards to users, and approve lasers for service use. This paper outlines current and projected use of lasers as battlefield tools, sensors, and weapons. In addition, it describes the USAF safety certification processes for weapon and non-weapon lasers, and measures taken to protect human safety of USAF and non-USAF personnel. Since perfect safety is impossible, the USAF safety oversight process seeks to balance personal safety with the needs of military operations. Technical measurements and analyses are used to inform a decisionmaking body that approves systems for use with appropriate safety constraints.

Keywords: lasers, Air Force safety, LSSRB, DEWCB, approval, exemption.
\end{abstract}

\section{Introduction}

Air Force safety is no accident! The Air Force has developed a culture of safety based on this motto, from the highest ranks right down to newly recruited Airmen. The Air Force embraces the idea of making sure each and every member is properly protected and safe while performing his or her duties. Whether it is on the battlefield or in garrison, protocols, policy, and guidance establish necessary steps to lead the way. The central organization that oversees Air Force safety is the Headquarters Air Force Safety Center (HQ AFSC). Members of this organization can get involved in various aspects of Air Force safety, but the primary focus of the following paper deals with the directed energy team. How the safety process works, what is available in the inventory, and who is involved are just a few questions that will be answered. 


\section{Air Force}

There is no question that the United States military devotes significant money and resources to develop state of the art weapons. The U.S. employs some of the most sophisticated systems in the world. What is less known or talked about in mainstream media is how much effort is involved in making sure the technology has adequate controls, procedures, and training before it is fielded. The safety process begins at the start of program development. It is a requirement for the program manager of any Department of Defense (DoD) program to provide basic safety information, with respect to the device or weapon, during initial stages of development and throughout its lifetime. The concept is safety from cradle to grave. This process is initiated by contacting the appropriate organizations within the Air Force.

\subsection{Organizations}

There is one major Air Force organization involved with providing safety for weapon systems. HQ AFSC is the overarching organization in charge of making sure weapons and devices are safe for Air Force personnel. Policy and guidance are constantly updated, reviewed, and processed to keep up with changing issues and technology. The Chief of Safety and a small office at the Pentagon directly support safety issues and disseminate safety guidance.

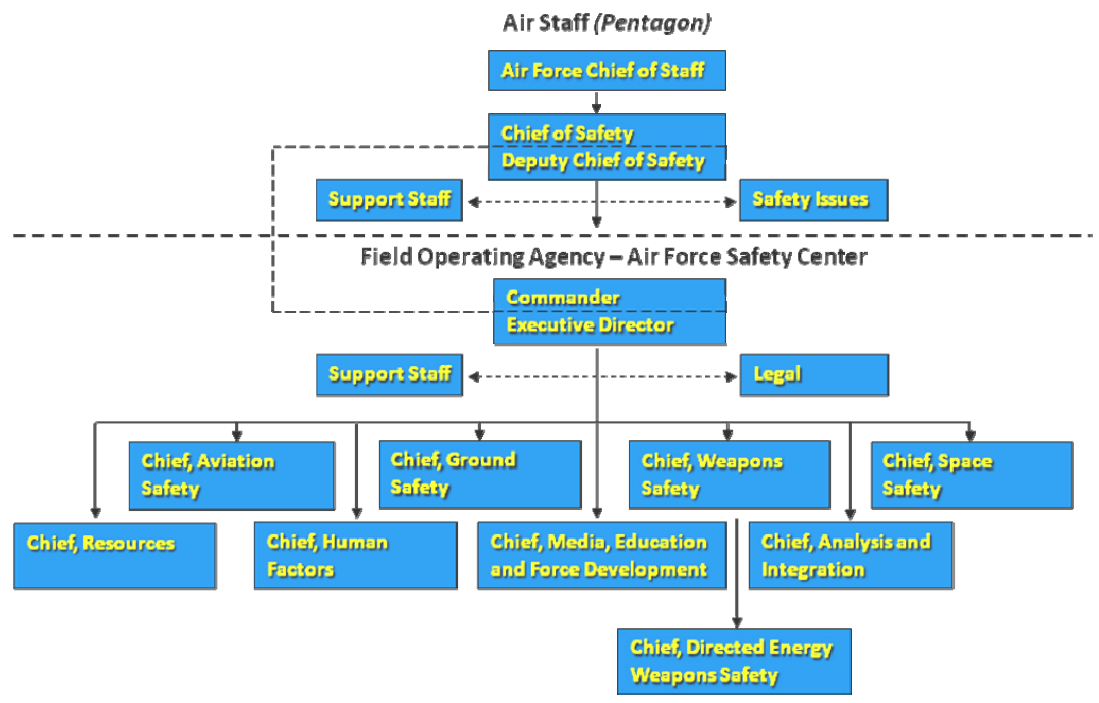

Figure 1: HQ AFSC structure.

HQ AFSC is the central authority for safety guidance, policy, analysis, and training for the Air Force. Another important organization exists when dealing with directed energy specifically. The Air Force Research Laboratory (AFRL) 
plays a significant role in developing technologies for the Air Force and analyzing health effects of Air Force weapons. The organization is charged with creating, sustaining, and implementing tools to aid national security. AFRL conducts research and supports efforts in system development and acquisition, testing, system safety, etc. under the directed energy directorate. The directed energy directorate provides research and development for directed energy devices and weapons. For the purpose of this paper, a closer look is given to the directed energy safety and health structure within AFRL, located in figure 2. The $711^{\text {th }}$ Human Performance Wing contains two branches in support of directed energy safety and technology assessment. Directed Energy devices as well as weapons are analyzed by the optical radiation (RHDO) and radiofrequency radiation (RHDR) branches, depending on system specifications. Figure 2 represents the organizational structure associated with directed energy safety and health under AFRL.

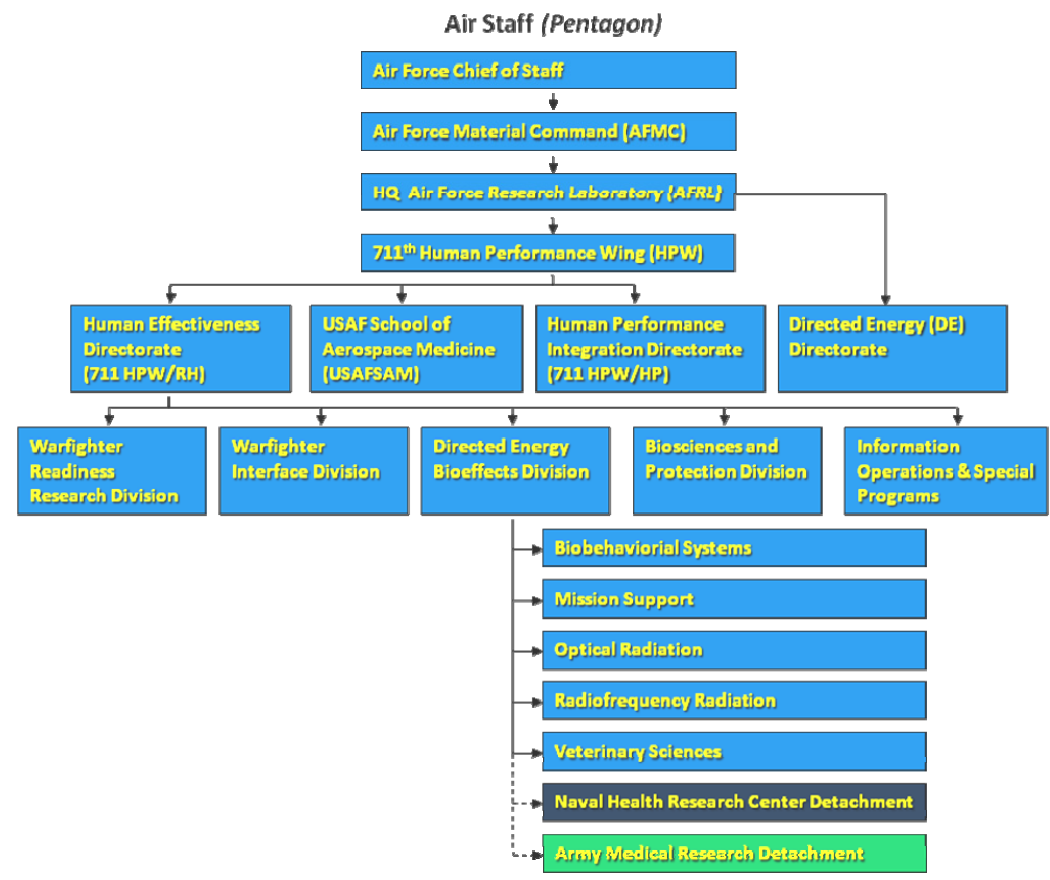

Figure 2: $\quad 711^{\text {th }} \mathrm{HPW}$ structure.

The optical radiation and radiofrequency radiation branches are part of a division devoted to testing, analyzing data, and developing a better understanding of biological effects related to directed energy weapons and devices. The belief is that knowing how a technology affects humans provides a safer environment for everyone. U.S. intentions are to utilize force only when necessary and to know the consequences related to the force applied for any situation. 


\section{Types of weapons}

Any situation involving national security can involve a number of weapon types, the broad spectrum being conventional and nuclear munitions. An emerging weapon type is Directed Energy (DE), the basis of this paper. The overall understanding of U.S. Air Force weapons provides a better base to build on for an in depth discussion of DE.

\subsection{Conventional}

There are many different types of conventional weapons in the Air Force inventory. Typical selections involve missiles, bombs, bullets, and supporting equipment. This type of weapon has a long history, and safety measures have been employed and understood for decades. That does not mean safety standards are less stringent. It simply means there is a more refined process for dealing with conventional munitions.

\subsection{Nuclear}

Compared to conventional weapons, there are few varieties of nuclear weapons. The nuclear weapon inventory consists of bombs and missiles. The Air Force also provides safety certification of nuclear support equipment, such as carts, tools, and trucks. This means every piece of equipment utilized for movement or maintenance, as well as the physical weapon, has to be safety certified and adhere to strict guidance related to the subject. This type of weapon is maintained but new developments have been limited because of post Cold War initiatives. Safety processes have been in place for decades, but not as long as conventional weapons.

\subsection{Directed energy weapons (DEW)}

A somewhat newer form of weapon is classified as directed energy. Most research and development in the DE field deals with lasers or radio-frequency transmitters. This type of weapon is largely based on theoretical understanding of electrical engineering principles because, unlike conventional and nuclear munitions, electronic beams and waves cannot be seen outside of the visible light portion of the electromagnetic spectrum. DEWs have instantaneous delivery and can be designed to be lethal or non-lethal. The Air Force conducts research and development for both laser and microwave DE weapons, but this paper will focus on lasers. Lasers can be found in the visible and invisible spectrum, anywhere between wavelengths of 200 and 1,000,000 nanometers. The Air Force is providing excellent data and safety analysis on existing systems to further these DE technologies. Lasers are a futuristic weapon that can limit collateral damage, and help minimize casualties when a country is faced with war. 
ELECTROMAGNETIC SPECTRUM

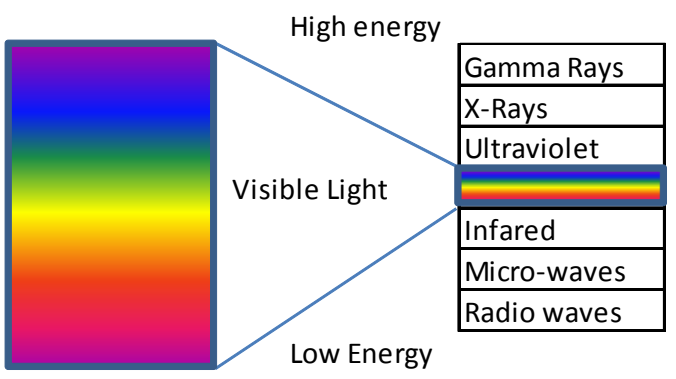

Figure 3: Electromagnetic spectrum.

\section{Safety}

All Air Force weapons go through a rigorous safety review from inception. Each of them has to meet a certification board to be approved for use. As Air Force use of DE increases, the more refined the approval process will become. There are currently two different safety approval processes performed by HQ AFSC. The first stems from the use of one type of non-weapon directed energy devices, lasers. The second is the approval of directed energy weapons. A device is any DE system that is not a weapon; to clarify, a weapon can be lethal or non-lethal. The distinct difference depends on the intended use of the system. The Air Force has implemented safety approval processes by viewing the DE system from these two perspectives. Air Force safety guidance [1-3] is based on DoD guidance [4] that implements US industrial standards [5] and federal regulations [6]. If a laser cannot comply with federal regulations while fulfilling its military mission, the board can exempt it from those regulations to allow purchase by a military organization.

\subsection{DE devices}

Directed energy devices consist of lasers, radars, radio transmitters, etc. The only DE devices that require approval by the U.S. Air Force are laser devices. Safety guidance for other DE devices (radio transmitters, radars, etc.) is well defined and adequate for protecting personnel. Laser technology has more significant hazards than other DE devices, thus a formal approval process was adopted. The word "device" is the key when approaching the approval process. Categories of use include rangefinders, designators, and illuminators. Since the laser is not intended to harm enemy personnel, equipment, or facilities, it is considered a device rather than a weapon. U.S. laser manufacturers have to adhere to Food and Drug Administration (FDA) regulations when producing a laser for occupational use, 21 CFR 1040.10 or 21 CFR 1040.11. The laser has to be safe for the general public operating it. Some FDA safety requirements, such as bright labels or warning lights, may not be compatible with military operations. For this reason, the Air Force and other U.S. services are allowed to 
exempt lasers from the normal Federal safety regulations and use militaryspecific safety controls. HQ AFSC issues exemptions to manufacturers. The Air Force has seen an increase in devices requiring approvals like this. HQ AFSC does not provide a laser approval for medical or industrial lasers, which must all be in compliance with FDA regulations.

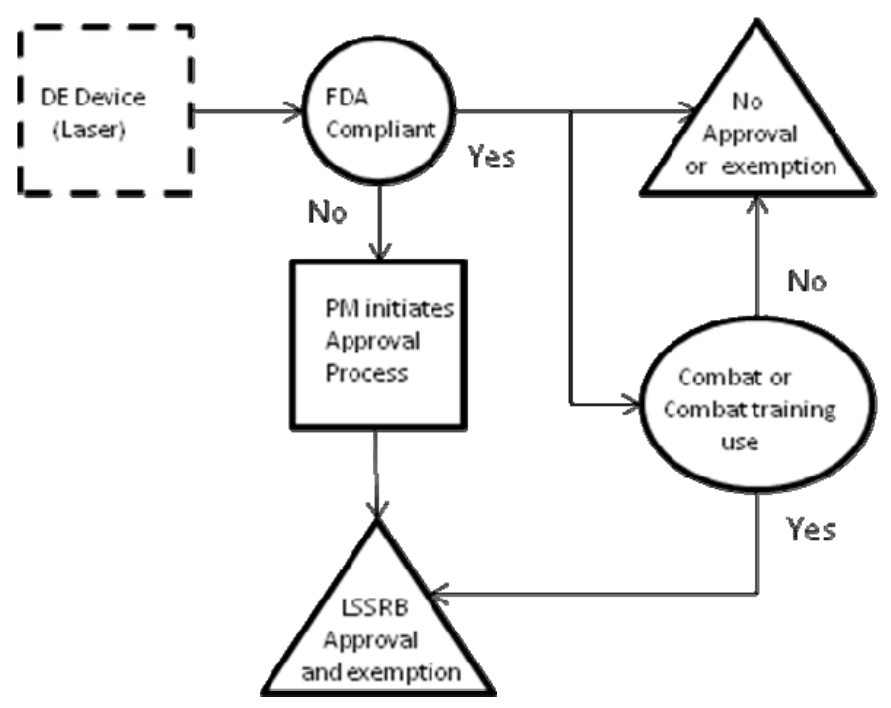

Figure 4: DE device process flow.

HQ AFSC requires implementation of the approval process for new lasers intended for combat or combat training use, or when modifications to existing lasers occur. The approval process begins when the Program Manager (PM) notifies the manufacturer that the system needs approval, or vice versa. The PM then contacts HQ AFSC for safety board approval initiation and the Human Effectiveness Directorate, Directed Energy Bio effects Division, Optical Radiation Branch (RHDO) for device assessment. RHDO, a subcomponent of AFRL, has the capability to analyze the laser for compliance with Federal regulations and Air Force standards. After the assessment is complete, information is passed to HQ AFSC for the Laser System Safety Review Board (LSSRB). HQ AFSC compiles all training, safety, and supporting documentation on the laser being reviewed then makes the information available for all LSSRB members. Each board member and technical specialist is notified of the posting for his/her review. The LSSRB is comprised of specialists in the field of lasers, law, medical, end-users, safety, major commands, and test and evaluation. Each evaluation looks at several laser criteria for safe, suitable, fielding. The list includes, but is not all inclusive: wavelength, power, divergence, controls, system stability, reflections, storage, maintenance, transportation, disposition, hazards (industrial, health, environmental, ancillary). Each specialty provides input to the board for a successful approval. HQ AFSC works with developers and purchasers to get approvals done in a timely manner 
while ensuring a thorough review of hazards. Once all Community of Practice (CoP) members have reviewed the laser, submitted comments, and the deadline has passed, modifications and/or corrections are made before the approval letter reaches the next review and approval level. The formal LSSRB approval letter is reviewed and signed by the chief of weapons safety, thus enabling and providing guidance for safe use of the DE device (laser).

\section{Laser System Safety Review Board Process (LSSRB)}

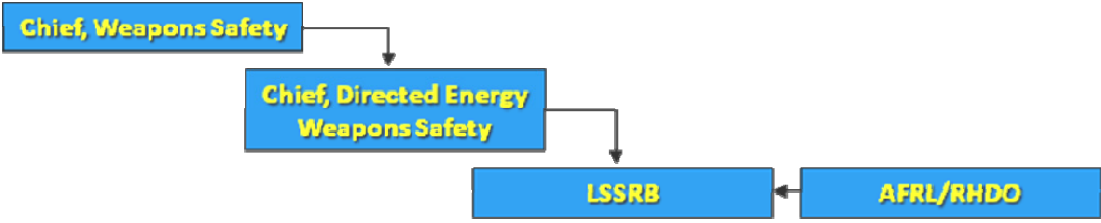

Figure 5: $\quad$ LSSRB structure.

\subsection{DE weapons}

A Directed Energy Weapon (DEW) takes a slightly different approval process path. DE weapons are primarily used to deny, disrupt, degrade, or destroy enemy equipment, facilities, or personnel. Examples of current and future use systems include Airborne Laser Test Bed, the GLARE MOUT laser dazzler, and the Active Denial System. Air Force guidance documents for DE, such as AFI 91-401, Directed Energy Weapons Safety, get reviewed and updated like other Air Force publications to stay current and relevant for present day issues. The instruction provides up to date guidance on formal and informal acquisition of DEW. The formal acquisition safety certification process for DEW is conducted in two phases. The certification phases are aligned with the overall program acquisition phases and decision milestones. Phase $\mathrm{I}$ is initiated sixty days after the formal acquisition program initiation (Milestone B) and entry into the System Development and Demonstration portion of the program. This phase notifies the safety community of the upcoming DEW and allows the PM to actively engage safety specialists in the early stages of DEW development. This is a key step in the development process. Early recognition of safety issues can result in correct, cost effective implementation of solutions. The PM is responsible for initiating and properly maintaining safety documentation for a DE weapon just as he/she does for a DE device or any new system. Phase II occurs after the decision (Milestone C) to commence the production and deployment portion of the program.

A DE weapon goes before the Directed Energy Weapons Certification Board (DEWCB). HQ AFSC is again responsible for compiling and disseminating all of the supporting documentation related to the DEW up for review. The DEWCB CoP is then loaded with all pertinent information and each member is notified and given a deadline to review and provide comments. The DE weapons board consists of experts in the safety, medical, surgeon general, 
weapons, and research fields. The DEWCB evaluates a system using many of the same criteria as the LSSRB, such as stray laser energy (SLE), hazards, power, user controls, stability, etc. Once the safety certification review board is complete, the approval letter is formalized. The Air Force chief of weapons safety is the final approving authority. A caveat to the process is significant deficiencies. Safety issues unveiled during the board review can lead to a statement of deficiencies from the chief of weapons safety, and disapproval of the DE weapon. The PM must implement solutions before re-submission to the approval board. HQ AFSC is committed to make the right decisions early to prevent mishaps later on in the process.

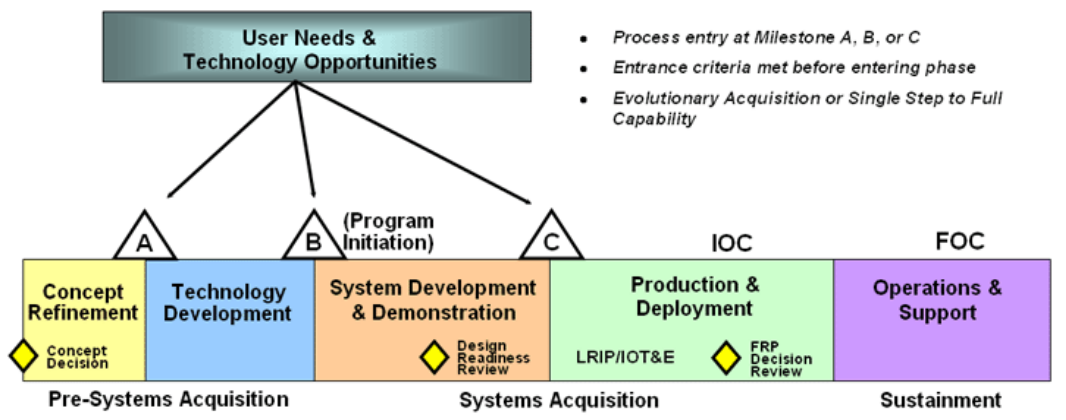

Figure 6: $\quad$ Formal acquisition process.

The informal acquisition process closely resembles the formal acquisition process. Research and development projects generally fall in to this process. There must be a safety review before the DEW can be used for operations or training, or demonstrated outside the U.S.A. The PM is still responsible for advising approval boards. One approval phase is conducted, and it is specifically tailored to the needs of the project. An informal acquisition program will have to meet certain requirements from the formal process to meet the demands of the project. The flexibility allows the informal process to fit any number of scenarios.

\section{Directed Energy Weapons Certification Board Process (DEWCB)}

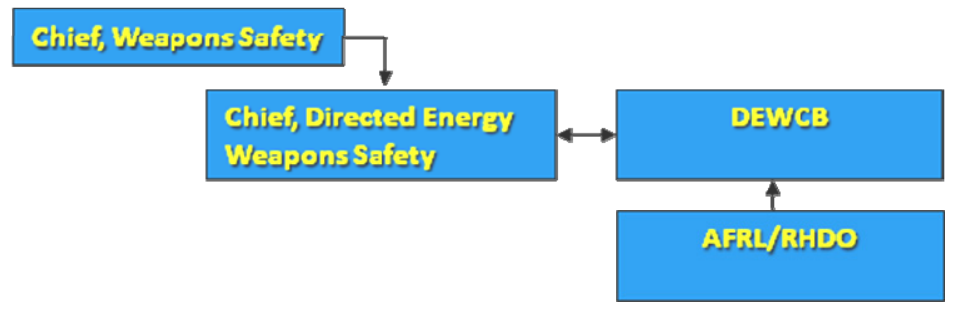

Figure 7: DEWCB structure. 
Modifications to existing DEW systems require re-verification. A system that has previously been identified as safe must be re-submitted to HQ AFSC for final approval. Re-verification requirements include but are not limited to physical, functional, and configuration changes. HQ AFSC reserves the right to accept the changes or return the system to the board to determine if safety certification should be continued or terminated.

\section{Conclusion}

As it stands right now, the Air Force has two well structured safety approval processes for DE devices and DE weapons. Increased usage of lasers and millimeter wave or microwave technology will undoubtedly allow the Air Force to refine these processes just as conventional weapon safety approval processes have done over the years. It is important to be flexible as new technologies such as acoustic and particle weaponry evolve. They will need safety oversight as well. From an international perspective, the U.S. government and researchers alike will continue to develop new DE devices and weapons, but with the right safety mindset and early integration, these Air Force safety processes will endure because of simplicity and applicability.

\section{References}

[1] Air Force Instruction (AFI) 91-401, Directed Energy Weapons Safety, Secretary of the Air Force, September 2008.

[2] Air Force Occupational Safety and Health Standard (AFOSH STD) 48-139, Laser Radiation Protection Program, Secretary of the Air Force, December 1999.

[3] Air Force Policy Directive (AFPD) 91-4, Directed Energy Weapons (DEW) Safety, Secretary of the Air Force, July 2003.

[4] Department of Defense Instruction (DoDI) 6055.15, DoD Laser Protection Program, Under Secretary of Defense, May 2007.

[5] American National Standards Institute (ANSI), American National Standard for Safe Use of Lasers, Z-136.1-2007, Orlando, FL, Laser Institute of America, 2007.

[6] Title 21, Code of Federal Regulations, Sections 1040.10 and 1040.11. 